\title{
Orthotropic transform for planar anisotropic elasticity and reduced dependence of elastic constants
}

\author{
By Wei YAng ${ }^{1}$ AND CHIEn-Ching M A ${ }^{2}$ \\ ${ }^{1}$ Department of Engineering Mechanics, Tsinghua University, \\ Beijing 100084 \\ ${ }^{2}$ Department of Mechanical Engineering, National Taiwan University, \\ Taipei 10617
}

Received 4 February 1997; accepted 16 September 1997

The resurgence in the literature on the reduced dependence of elastic constants for damaged materials and composites has been largely inspired by the exploration of the stress invariance under a modulus shift by Cherkeav and others in 1992 (henceforth referred to as the CLM shift) in planar elasticity. However, a CLM shift can at most reduce the dependence of two elastic constants. The present work devises an approach that combines the CLM shift and an orthotropic transform. As a result, the governing equation of a general planar anisotropic solid only contains a single dimensionless elastic constant (denoted by $\left|c_{2}^{*}\right|$ in this paper) in the transformed coordinates. The damage compliance of a solid with holes can be expressed explicitly through a function depending only on $\left|c_{2}^{*}\right|$. If an elastic composite consists of a matrix and inclusions of aligned anisotropy, its overall compliance can be expressed through a function of three combinative parameters, namely $\left|c_{2}^{*}\right|$ and two Dundurs parameters. Furthermore, a configuration invariant transform is found for layered materials of aligned anisotropy. The stress analysis of such configurations only involves four elastic constants.

Keywords: orthotropic transform; planar anisotropy; elastic constants; holes; inclusions; layered materials

\section{Introduction}

The analysis in anisotropic elasticity is tedious by the presence of many elastic constants. It is desirable from both theoretical and practical points of view to reduce the dependence of elastic constants before the actual analysis on a boundary value problem. Significant reduction of the problem was achieved by the CLM (Cherkeav et al. 1992) shift, which effectively eliminates two elastic constants in most cases. Dundurs \& Markenscoff (1993) generalized the uniform CLM shift to include a linear alternation of the elastic compliance. Moran \& Gosz (1994) extended the shift to situations concerning a variety of interface conditions. The study of stress invariance can be traced back almost a century to the pioneer work of Michell, who pointed out the amazing result that the two-dimensional stress analysis for a multiply connected isotropic solid does not depend on the elastic constants if self-equilibrium traction is prescribed on each closed contours. In the context of in-plane deformation of an isotropic solid with holes and non-contacting microcracks, the theorem

Proc. R. Soc. Lond. A (1998) 454, 1843-1855

Printed in Great Britain 
of Michell was elaborated to show that the damage compliance $\boldsymbol{H}$ (defined as the difference between the overall compliance and the matrix compliance) is independent of the matrix elastic constants (see Day et al. 1992; Cherkaev et al. 1992; Thorpe \& Jasiuk 1992; Christensen 1993; Zheng \& Hwang 1997). For a planar composite with two isotropic phases, Zheng \& Hwang (1996) reported that $\boldsymbol{H}$ only depends on two dimensionless parameters (with the famous Dundurs parameters as one possible set of choices), under a variety of interface relations discussed by Moran \& Gosz (1994).

A CLM shift can at most reduce the dependence of two elastic constants. We refer to the works of Dundurs \& Markenscoff (1993), Moran \& Gosz (1994) and Ting (1995) as examples of two constants reduction for planar anisotropic materials, composites and bimaterials. The problems of planar anisotropic elasticity, however, contain six elastic constants in a single material phase. A reduction of two elastic constants barely makes the problem tractable. The present work explores an approach that further reduces the dependence of elastic constants. We recapitulate the twodimensional anisotropic elasticity in the next section, assuming the absence of body force and inertia term. Section 3 introduces an affine transform. In determining the transform, we reduce the general planar anisotropy to a special class of orthotropy in the transformed coordinates. We henceforth term the transform an orthotropic transform. Combined with the CLM shift, we are able to reduce a self-equilibrated traction boundary value problem to the one which only contains a single dimensionless elastic constant (denoted by $\left|c_{2}^{*}\right|$ ) in the transformed coordinates. The remaining part of the paper provides three applications of orthotropic transform. Section 4 shows that the damage compliance of a planar anisotropic solid with voids can be expressed explicitly through a function of $\left|c_{2}^{*}\right|$. Two-phase elastic composites with aligned anisotropy are analysed in $\S 5$. Although eight elastic constants are involved in this material system, we are able to express the overall compliance of the composite by a function of three combinative parameters: $\left|c_{2}^{*}\right|$ and two Dundurs parameters. Section 6 considers the stress analysis for aligned anisotropic bimaterials with straight interfaces, covering the configurations of interfacial fracture and the stress analysis of layered materials. Only four dimensionless elastic constants stem out after a transform which reverses the configuration; they are $\left|c_{2}^{*}\right|$, two Dundurs parameters and the rotation angle of interface by orthotropic transform.

\section{Formulation of planar anisotropic elasticity}

Consider two-dimensional plane strain deformation. The approach developed here can be extended to the generalized plane stress state. The in-plane Cartesian coordinates are denoted by $x_{1}$ and $x_{2}$, and their unit base vectors by $\boldsymbol{e}_{1}$ and $\boldsymbol{e}_{2}$. In the absence of body force and inertia term, the two-dimensional equilibrium equation is

$$
\sigma_{i j, i}=0 .
$$

Throughout this work, the indices of a vector or a tensor range from 1 to 2 , and the summation rule applies for repeated indices. A subscripted prime abbreviates for partial differentiation. The inclusion of body force into this formulation can be pursued by a body force potential, as treated in Green \& Zerna (1960). The inclusion of the inertial term might be possible in the steady-state case, or, in the vicinity of a propagating crack tip, as discussed by Yang et al. (1991). The equilibrium equation (2.1) can be satisfied by introducing an Airy stress function $\Phi\left(x_{1}, x_{2}\right)$ such

Proc. R. Soc. Lond. A (1998) 
that

$$
\sigma_{i j}=J_{i j k l} \Phi_{, k l},
$$

where tensor $\boldsymbol{J}$ (bold letters represent the whole tensors) in (2.2) can be regarded as a fourth-order rotation tensor, as interpreted by Cherkaev et al. (1992), and is given by

$$
\boldsymbol{J}=\mathbf{1} \otimes \mathbf{1}-\boldsymbol{I},
$$

where symbol $\otimes$ denotes tensorial product, and $\mathbf{1}$ and $\boldsymbol{I}$ are the second- and fourthorder identity tensors. With the help of $\boldsymbol{J}$, one can cast the two-dimensional compatibility equation into the following form:

$$
J_{i j k l} \varepsilon_{i j, k l}=0 .
$$

The two-dimensional linear elastic constitutive relation is given by

$$
\varepsilon_{i j}=S_{i j k l} \sigma_{k l},
$$

where $\boldsymbol{S}$ characterizes the fourth-order compliance tensor. For a planar anisotropic solid, a general presentation (see, for example, Zheng \& Hwang 1996, 1997) of $\boldsymbol{S}$ is

$$
\boldsymbol{S}=(1 / E)\left\{\boldsymbol{I}-\nu \boldsymbol{J}+\frac{1}{2}\left(\mathbf{1} \otimes \boldsymbol{D}_{2}+\boldsymbol{D}_{2} \otimes \mathbf{1}\right)+\boldsymbol{D}_{4}\right\},
$$

where $\boldsymbol{D}_{2}$ and $\boldsymbol{D}_{4}$ are the second- and fourth-order deviatoric tensors, and are completely symmetric and traceless. Scalars $E$ and $\nu$ represent the average Young's modulus and the average Poisson's ratio for a planar anisotropic solid in a plane strain deformation. The first two terms in (2.6) describe the average isotropic compliance, whose dependence can be reduced by a CLM type shift. The last two terms featuring deviatoric tensors $\boldsymbol{D}_{2}$ and $\boldsymbol{D}_{4}$ describe the anisotropy of the material.

To get explicit representations of $\boldsymbol{D}_{2}$ and $\boldsymbol{D}_{4}$, a complex base $\boldsymbol{Z}_{n}$ for a tensor of the $n$th order is introduced

$$
\boldsymbol{Z}_{n}=\boldsymbol{e}_{z} \otimes \boldsymbol{e}_{z} \otimes \cdots \otimes \boldsymbol{e}_{z} \quad n \text { times, } \quad \boldsymbol{e}_{z}=\boldsymbol{e}_{1}+\mathrm{i} \boldsymbol{e}_{2},
$$

where $\mathrm{i}=\sqrt{-1}$. Facilitated by this base vector, the completely symmetric and traceless tensors $\boldsymbol{D}_{2}$ and $\boldsymbol{D}_{4}$ are expressed as

$$
\boldsymbol{D}_{2}=\operatorname{Re}\left\{\bar{c}_{2} \boldsymbol{Z}_{2}\right\}, \quad \boldsymbol{D}_{4}=\operatorname{Re}\left\{\bar{c}_{4} \boldsymbol{Z}_{4}\right\},
$$

where the overbars denote complex conjugate, and the dimensionless complex constants $c_{2}$ and $c_{4}$ are given by four real constants $a_{2}, a_{4}, b_{2}, b_{4}$, where

$$
c_{2}=a_{2}+\mathrm{i} b_{2}, \quad c_{4}=a_{4}+\mathrm{i} b_{4} .
$$

Substituting (2.8) and (2.7) into (2.6), we arrive at the following six-parameter presentation for the elastic compliance of a planar anisotropic solid:

$$
\boldsymbol{S}=(1 / E) \operatorname{Re}\left\{\boldsymbol{I}-\nu \boldsymbol{J}+\frac{1}{2} \bar{c}_{2}\left(\mathbf{1} \otimes \boldsymbol{e}_{z} \otimes \boldsymbol{e}_{z}+\boldsymbol{e}_{z} \otimes \boldsymbol{e}_{z} \otimes \mathbf{1}\right)+\bar{c}_{4} \boldsymbol{e}_{z} \otimes \boldsymbol{e}_{z} \otimes \boldsymbol{e}_{z} \otimes \boldsymbol{e}_{z}\right\}
$$

The anisotropy of the solid is solely characterized by two complex constants $c_{2}$ and $c_{4}$. A tetratropic solid corresponds to the case of $c_{2}=0$, and an orthotropic solid corresponds to the case of $b_{2}=b_{4}=0$ if the coordinate axes are aligned with the orthotropic axes. Planar isotropy requires both $c_{2}$ and $c_{4}$ to vanish. Detailed discussion for material symmetry can be found from Zheng \& Hwang (1996, 1997).

Proc. R. Soc. Lond. A (1998) 
For example, the in-plane tensile modulus $E(\theta)$ in a direction which forms an angle $\theta$ with the $x_{1}$-axis has the following form:

$$
E / E(\theta)=1+\operatorname{Re}\left\{\bar{c}_{2} \mathrm{e}^{2 \mathrm{i} \theta}+\bar{c}_{4} \mathrm{e}^{4 \mathrm{i} \theta}\right\} .
$$

Apparently, $c_{4}$ describes the tetratropic response of the solid, and refers to the response which only has a two-fold symmetry with respect to $\theta$. The positive-definiteness of the compliance tensor requires

$$
\left.\begin{array}{c}
1+a_{2}+a_{4}>0, \quad \frac{1}{2}(1+\nu)+a_{4}>\frac{a_{2}^{2}}{2(1-\nu)}, \quad \nu<1, \\
\left(\frac{1}{2}(1+\nu)+a_{4}-\frac{a_{2}^{2}}{2(1-\nu)}\right)\left(\frac{1}{2}(1+\nu)-a_{4}\right)>\left(b_{2}+b_{4}\right)^{2} .
\end{array}\right\}
$$

Equations (2.2), (2.4) and (2.5) give rise to a governing equation of the Airy stress function,

$$
S_{i j k l}^{\perp} \Phi_{, i j k l}=0,
$$

where

$$
\begin{aligned}
\boldsymbol{S}^{\perp}=\boldsymbol{J}: \boldsymbol{S}: \boldsymbol{J}=(1 / E) \operatorname{Re}\left\{\boldsymbol{I}-\nu \boldsymbol{J}-\frac{1}{2} \bar{c}_{2}\left(\mathbf{1} \otimes \boldsymbol{e}_{z} \otimes \boldsymbol{e}_{z}+\boldsymbol{e}_{z} \otimes \boldsymbol{e}_{z} \otimes \mathbf{1}\right)\right. \\
\left.+\bar{c}_{4} \boldsymbol{e}_{z} \otimes \boldsymbol{e}_{z} \otimes \boldsymbol{e}_{z} \otimes \boldsymbol{e}_{z}\right\}
\end{aligned}
$$

bears the physical significance of $\boldsymbol{S}$ rotated (through the fourth-order rotation tensor $\boldsymbol{J})$ by $90^{\circ}$, similar to the interpretation by Cherkaev et al. (1992) for the isotropic case. The geometric meaning of rotation becomes transparent in (2.11), where a rotation of $90^{\circ}$ leaves $c_{4}$ unchanged but replaces $c_{2}$ by its negative value, as occurred in (2.14).

The governing equations (2.13) and (2.14) can be put into a compact form by introducing complex variable $z=x_{1}+\mathrm{i} x_{2}$. Regarding $\Phi$ as a function of $z$ and its complex conjugate, one writes the governing equation in complex form as

$$
\operatorname{Re}\left\{\left(\frac{\partial^{2}}{\partial \bar{z}^{2}}-c_{2} \frac{\partial^{2}}{\partial z \partial \bar{z}}+c_{4} \frac{\partial^{2}}{\partial z^{2}}\right) \frac{\partial^{2} \Phi}{\partial z^{2}}\right\}=0
$$

As implicated by the CLM shift, the dependence on the isotropic material properties $E$ and $\nu$ naturally drops out from the governing equation.

\section{Orthotropic transform}

We outline in this section a procedure that drastically simplifies the governing equation. Consider the following variable transform:

$$
\zeta=z+\gamma \bar{z}
$$

where $\gamma=\gamma_{1}+\mathrm{i} \gamma_{2}$ denotes a complex constant yet to be determined. The transform (3.1) is affine, and maps parallel straight lines in the $z$-plane to parallel straight lines in the $\zeta$-plane. Thus, (3.1) will not complicate the geometry in the transformed coordinates for problems involving straight interfaces, layered structures and straight crack arrays. The slope of a straight line in the plane increases with the slope of its image in the $z$-plane if $|\gamma|<1$, and decreases with the slope of its image in the $z$-plane if $|\gamma|>1$. The case of $|\gamma|=1$ maps the entire $z$-plane to a single line in the 
$\zeta$-plane; this singular case has therefore to be avoided. To reverse the sense of the coordinates in the transformed plane, it is preferable to choose $|\gamma|<1$. We will show in the sequel that the choice of $|\gamma|<1$ is always possible in the present scheme.

We now note that the Airy stress function depends on the transformed variables $\zeta$ and $\bar{\zeta}$. By straightforward algebra, the governing equation (2.15) is transformed to

$$
\operatorname{Re}\left\{\left(\frac{\partial^{2}}{\partial \bar{\zeta}^{2}}-c_{2}^{*} \frac{\partial^{2}}{\partial \zeta \partial \bar{\zeta}}+c_{4}^{*} \frac{\partial^{2}}{\partial \zeta^{2}}\right) \frac{\partial^{2} \Phi}{\partial \zeta^{2}}\right\}=0,
$$

where

$$
c_{2}^{*}=\frac{\tilde{c}_{2}}{a}, \quad c_{4}^{*}=\frac{\tilde{c}_{4}}{a}
$$

and

$$
\left.\begin{array}{rl}
\tilde{c}_{2} & =c_{2}(1+3 \gamma \bar{\gamma})+\bar{c}_{2} \gamma^{2}(3+\gamma \bar{\gamma})-4 c_{4} \bar{\gamma}-4 \bar{c}_{4} \gamma^{3}-4 \gamma(1+\gamma \bar{\gamma}), \\
\tilde{c}_{4} & =c_{4}-c_{2} \gamma+2 \gamma^{2}-\bar{c}_{2} \gamma^{3}+\bar{c}_{4} \gamma^{4} \\
a & =1+4 \gamma \bar{\gamma}+\gamma^{2} \bar{\gamma}^{2}-3(1+\gamma \bar{\gamma}) \operatorname{Re}\left(\bar{c}_{2} \gamma\right)+6 \operatorname{Re}\left(\bar{c}_{4} \gamma^{2}\right) .
\end{array}\right\}
$$

Clearly $a$ is real. To proceed, we determine the transformation parameter $\gamma$ by

$$
c_{4}-c_{2} \gamma+2 \gamma^{2}-\bar{c}_{2} \gamma^{3}+\bar{c}_{4} \gamma^{4}=0 .
$$

If $\gamma$ is a root of the quartic equation (3.5), so is $1 / \bar{\gamma}$. The four roots of (3.5) have the form $\left(\gamma, \gamma^{\prime}, 1 / \bar{\gamma}, 1 / \bar{\gamma}^{\prime}\right)$. Thus, there are always two roots whose amplitudes are less than one. We will choose any one of these two roots in the transformation (3.1), which leads to the following simplifications:

$$
c_{4}^{*}=0, \quad \tilde{c}_{2}=(1-\gamma \bar{\gamma})\left[4\left(\gamma+c_{4} / \gamma\right)-3 c_{2}-\bar{c} \gamma^{2}\right] .
$$

If one further performs a rotational transform,

$$
z^{*}=x_{1}^{*}+\mathrm{i} x_{2}^{*}=\mathrm{e}^{\mathrm{i} \omega} \zeta, \quad \text { with } \quad \omega=-\frac{1}{2} \arg \tilde{c}_{2},
$$

then the governing equation (3.2) is reduced to

$$
\left\{\nabla^{* 2}-\left|c_{2}^{*}\right|\left(\frac{\partial^{2}}{\partial x_{1}^{* 2}}-\frac{\partial^{2}}{\partial x_{2}^{* 2}}\right)\right\} \nabla^{* 2} \Phi=0,
$$

where $\nabla^{* 2}$ is the Laplace operator in the transformed coordinates $x_{i}^{*}$. All quantities labelled by an asterisk will be referred to the coordinates $x_{i}^{*}$. The coordinate transform defined by (3.1), (3.5) and (3.7) is termed orthotropic transform in this paper. After an orthotropic transform, the four real anisotropic constants reduce to a single real constant in the governing equation (3.8). The orthotropic transform is summarized by

$$
x_{i}^{*}=\Omega_{i j} x_{j}, \quad \text { with } \quad \Omega_{i j}=\left[\begin{array}{rr}
\cos \omega & -\sin \omega \\
\sin \omega & \cos \omega
\end{array}\right]\left[\begin{array}{cc}
1+\gamma_{1} & -\gamma_{2} \\
\gamma_{2} & 1-\gamma_{1}
\end{array}\right] .
$$

The transformed expressions of traction and stresses can be obtained through the conventional transformation formulas for vectors and the second-order tensors,

$$
T_{1}^{*}=\Omega_{i k}^{-1} T_{k}, \quad \sigma_{i j}^{*}=\Omega_{i k}^{-1} \Omega_{j l}^{-1} \sigma_{k l},
$$

with

$$
\Omega_{i j}^{-1}=\frac{1}{1-\gamma \bar{\gamma}}\left[\begin{array}{cc}
1-\gamma_{1} & \gamma_{2} \\
-\gamma_{2} & 1+\gamma_{1}
\end{array}\right]\left[\begin{array}{rr}
\cos \omega & \sin \omega \\
-\sin \omega & \cos \omega
\end{array}\right]
$$

Proc. R. Soc. Lond. A (1998) 
The compliance tensor in the transformed coordinates is given by

$$
S_{i j k l}^{*}=\Omega_{i p} \Omega_{j q} \Omega_{k r} \Omega_{l s} S_{p q r s}=\left(1 / E^{*}\right) \operatorname{Re}\left\{I_{i j k l}-\nu^{*} J_{i j k l}+\frac{1}{2} \bar{c}_{2}^{*}\left(\delta_{i j} \xi_{k} \xi_{l}+\xi_{i} \xi_{j} \delta_{k l}\right)\right\},
$$

where $\xi_{1}=1$ and $\xi_{2}=\mathrm{i}$, and $\delta_{i j}$ is the Kronecker delta. The derivation towards the last step in (3.12) can be carried out as follows. One writes down the general form of $\boldsymbol{S}^{*}$ in the transformed coordinates, which assumes a similar form as the $\boldsymbol{S}$ expression in (2.10), but is projected in the $x_{i}^{*}$ coordinates. By this expression of $\boldsymbol{S}^{*}$, the governing equation for Airy stress function can be constructed. Comparing it with (3.8), one determines the parameters in $\boldsymbol{S}^{*}$ exactly as in the second expression of (3.12). The form of (3.12) reveals that in the $x_{i}^{*}$ coordinates, the response of a general planar anisotropic material is not only orthotropic, but also maintains an 'isotropic relation' among the average Young's modulus, shear modulus and Poisson's ratio:

$$
\mu^{*} \equiv \frac{1}{2 S_{1212}^{*}}=\frac{E^{*}}{2\left(1+\nu^{*}\right)} .
$$

The average Young's modulus and Poisson's ratio in the transformed coordinates can be evaluated from (3.12) and (3.11) after considerable algebra:

$$
\left.\begin{array}{rl}
E / E^{*} & =(1+\gamma \bar{\gamma})^{2}+2 \gamma_{1}^{2}\left(1+\gamma_{2}^{2}\right)+3 \Theta, \\
\nu^{*} & =\frac{\nu(1+\gamma \bar{\gamma})^{2}-2(1+2 \nu) \gamma_{1}^{2}\left(1+\gamma_{2}^{2}\right)-\Theta}{(1+\gamma \bar{\gamma})^{2}+2 \gamma_{1}^{2}\left(1+\gamma_{2}^{2}\right)+3 \Theta}, \\
\Theta & =\gamma_{1} \operatorname{Re}\left\{\left(1+\mathrm{i} \gamma_{2}\right)\left[(1+\gamma \bar{\gamma}) c_{2}+2 \gamma_{1} c_{4}\left(1+\mathrm{i} \gamma_{2}\right)\right]\right\} .
\end{array}\right\}
$$

We conclude this section by classifying the situations convertible to an isotropic formulation. Two restrictions among the elastic constants were found by Chiang (1994) for an isotropic formulation. In the present notation, they are

$$
\left(\frac{b_{2}-2 b_{4}}{b_{2}+2 b_{4}}\right)^{2}=\frac{1+a_{2}+a_{4}}{1-a_{2}+a_{4}}, \quad \pm 2 \sqrt{\frac{1+a_{2}+a_{4}}{1-a_{2}+a_{4}}}+\left(\frac{b_{2}-2 b_{4}}{b_{2}+2 b_{4}}\right)^{2}=\frac{2-6 a_{4}}{1-a_{2}+a_{4}} .
$$

The restrictions (3.15) can be replaced by requiring that $\tilde{c}_{2}$ in (3.6) vanishes. Namely,

$$
4\left(\gamma+c_{4} / \gamma\right)=3 c_{2}+\bar{c}_{2} \gamma^{2}
$$

The combination of (3.5) and (3.16) will give restrictions similar to (3.15) for an isotropic formulation. For the special case of a tetratropic material $\left(c_{2}=0\right)$, it is straightforward to show from either (3.15) or (3.16) that an isotropic response can never be realized in the transformed coordinates if $c_{4}$ is non-zero. For an orthotropic material $\left(b_{2}=b_{4}=0\right)$, an isotropic response in the transformed coordinates is possible only when $a_{2}^{2}=8 a_{4}\left(1-a_{4}\right)$, as can be found from either (3.15) or (3.16). In all the discussions above, the elastic constants should obey the restrictions (2.12) for the positive definiteness of the strain energy.

\section{Overall compliance for planar anisotropic solids with voids}

In this section we start to illustrate the power of orthotropic transform in reduced dependence on elastic constants. This section investigates a planar anisotropic solid 
with many cylindrical voids, with attention being focused on the overall compliance. Consider a sufficiently large continuum element with $N$ voids inside. The matrix of the continuum element occupies a region $\Omega$ of area $A$, bounded by the exterior boundary $\partial \Omega$ and the void surfaces $\Gamma_{\alpha}, \alpha=1,2, \ldots, N$. The traction on any of those boundaries is either zero or self-equilibrated, so that Michell's theorem applies. Under an orthotropic transform defined in the preceding section, the region $\Omega$, the area $A$, the exterior boundary $\partial \Omega$ and the void surfaces $\Gamma_{\alpha}$ are transformed to $\Omega^{*}$, $A^{*}, \partial \Omega^{*}$ and $\Gamma_{\alpha}^{*}$, respectively. The transformed traction remains zero along $\Gamma_{\alpha}^{*}$, and self-equilibrium along $\partial \Omega^{*}$. According to (3.10), stresses $\sigma_{i j}^{\infty}$ sustained along the exterior boundary $\partial \Omega$ are converted to $\sigma_{i j}^{\infty *}$ near $\partial \Omega^{*}$. Within the transformed domain $\Omega^{*}$, the compliance tensor assumes the simple three-parameter presentation (3.12). Parallel to the derivation for an isotropic material by Zheng \& Hwang (1997), the strain energy stored in $\Omega^{*}$ can be evaluated as

$$
U=\frac{1}{2} \int_{\Omega^{*}} \sigma_{i j}^{*} S_{i j k l}^{*} \sigma_{k l}^{*} \mathrm{~d} \Omega .
$$

The transformed stresses relate to the Airy stress function in a form similar to (2.2), except that the derivatives are taken with respect to the transformed coordinates. Using this expression and manipulating, one has

$$
\begin{aligned}
\sigma_{i j}^{*} S_{i j k l}^{*} \sigma_{k l}^{*} & =S_{i j k l}^{\perp *} \Phi_{, i^{*} j^{*}} \Phi_{, k^{*} l^{*}} \\
& =\frac{1}{E^{*}}\left\{\Phi_{, i^{*} j^{*}} \Phi_{, i^{*} j^{*}}+2 \nu^{*}\left(\Phi_{, 1^{*} 2^{*}}^{2}-\Phi_{, 1^{*} 1^{*}} \Phi_{, 2^{*} 2^{*}}\right)-\left|c_{2}^{*}\right|\left(\Phi_{, 1^{*} 1^{*}}^{2}-\Phi_{, 2^{*} 2^{*}}^{2}\right)\right\}
\end{aligned}
$$

When substituted into (4.1), the second term in (4.2) can be converted to a loop integral along the exterior boundary (Zheng \& Hwang 1997),

$$
2 \int_{\Omega^{*}}\left(\Phi_{, 1^{*} 2^{*}}^{2}-\Phi_{, 1^{*} 1^{*}} \Phi_{, 2^{*} 2^{*}}\right) \mathrm{d} \Omega=e_{i k} \sigma_{i j}^{\infty *} \oint_{\partial \Omega^{*}} P_{k}^{*} n_{j}^{*} \mathrm{~d} s,
$$

where $e_{i j}$ denotes the two-dimensional permutation symbol, $n_{j}^{*}$ the unit outward normal of $\partial \Omega^{*}$, and $P_{k}^{*}(s)$ the resultant traction along an arc measured by the arc length $s$. The traction free boundary conditions along the void surfaces $\Gamma_{\alpha}^{*}$ and the traction equilibrium boundary condition along the exterior boundary $\partial \Omega^{*}$ have been used in the above derivation, those conditions are instrumental in light of the Michell's restriction for stress invariance.

An alternative way to evaluate the strain energy of that continuum element is through macroscopically averaged quantities. Denote $\left\langle\boldsymbol{S}^{*}\right\rangle$ as the equivalent overall compliance tensor of the continuum element in the transformed coordinates. The presence of voids makes $\left\langle\boldsymbol{S}^{*}\right\rangle$ more compliant than $\boldsymbol{S}^{*}$. The damage compliance $\boldsymbol{H}$ is defined as the difference between the overall compliance and the matrix compliance. In the transformed coordinates, the above definition reads

$$
\left\langle\boldsymbol{S}^{*}\right\rangle=\boldsymbol{S}^{*}+\boldsymbol{H}^{*} \text {. }
$$

The strain energy stored in that element is given by

$$
\begin{aligned}
U= & \frac{1}{2} A^{*}\left(S_{i j k l}^{*}+H_{i j k l}^{*}\right) \sigma_{i j}^{\infty *} \sigma_{k l}^{*} \\
= & \frac{A^{*}}{2 E^{*}}\left[\sigma_{i j}^{\infty *} \sigma_{i j}^{\infty *}+\left|c_{2}^{*}\right|\left(\sigma_{11}^{\infty *}-\sigma_{22}^{\infty * 2}\right)\right] \\
& \quad+\frac{1}{2} A^{*} H_{i j k l}^{*} \sigma_{i j}^{\infty *} \sigma_{k l}^{\infty *}+\frac{\nu^{*}}{2 E^{*}} e_{i k} \sigma_{i j}^{\infty *} \oint_{\partial \Omega^{*}} P_{k}^{*} n_{j}^{*} \mathrm{~d} s .
\end{aligned}
$$

Proc. R. Soc. Lond. A (1998) 
The procedure leading to the second step of (4.5) is parallel to the derivations by Zheng \& Hwang (1997). Combining (4.1)-(4.3) and (4.5), we obtain

$$
\begin{aligned}
E^{*} H_{i j k l}^{*} \sigma_{i j}^{\infty *} \sigma_{k l}^{\infty *}=\frac{1}{2 A^{*}} & \left\{\int_{\Omega^{*}} \Phi_{, i^{*} j^{*}} \Phi_{, i^{*} j^{*}} \mathrm{~d} \Omega-\sigma_{i j}^{\infty *} \sigma_{i j}^{\infty *}\right. \\
& \left.-\left|c_{2}^{*}\right|\left[\int_{\Omega^{*}}\left(\Phi_{, 1^{*} 1^{*}}^{2}-\Phi_{, 2^{*} 2^{*}}^{2}\right) \mathrm{d} \Omega-\left(\sigma_{11}^{\infty * 2}-\sigma_{22}^{\infty * 2}\right)\right]\right\} .
\end{aligned}
$$

The linearity of the problem renders the Airy stress function $\Phi$ a linear combination of $\sigma_{i j}^{\infty *}$, and consequently the right-hand side of (4.6) would be a quadratic form of $\sigma_{i j}^{\infty *}$. Moreover, the stress function $\Phi$ depends on the material parameters only through $\left|c_{2}^{*}\right|$, and the same conclusion holds for the right-hand side of (4.6). We then have

$$
E^{*} H_{i j k l}^{*}=\Sigma_{i j k l}^{*}\left(\left|c_{2}^{*}\right|, g^{*}\right)
$$

where $g^{*}$ symbolizes the dependence on the void geometry in the transformed coordinates. We frequently lack detailed information of void geometry. Most void measurements are made for the volume fraction (or the area fraction for a plane strain case) $f$ of the voids. We note that the area fraction of the voids reverses under an orthotropic transform. If the damage compliance depends on the void geometry only through the area fraction of the voids, relation (4.7) can be reduced to

$$
E^{*} H_{i j k l}^{*}=\Sigma_{i j k l}^{*}\left(\left|c_{2}^{*}\right|, f\right) .
$$

We obtain the remarkable result that the transformed damage compliance of a general planar anisotropic material, when multiplied by $E^{*}$ defined in (3.14), only depends on a single dimensionless material parameter group $\left|c_{2}^{*}\right|$.

Combining (4.4) and (4.8), and transforming back, one obtains the overall compliance tensor in the $x_{1}-x_{2}$ coordinates,

$$
\left\langle S_{i j k l}\right\rangle=S_{i j k l}+\left(1 / E^{*}\right) \Omega_{p i}^{-1} \Omega_{q j}^{-1} \Omega_{r k}^{-1} \Omega_{s l}^{-1} \Sigma_{p q r s}^{*}\left(\left|c_{2}^{*}\right|, f\right),
$$

where the transformed average Young's modulus $E^{*}$ is given in (3.14), and the inverse transform matrix $\Omega_{p i}^{-1}$ is given in (3.11). Expression (4.9) prescribes the structure for the elastic constant dependence of overall compliance. For any micromechanics evaluations on the overall compliance of planar anisotropic solids with voids, the form of (4.9) can serve as a consistency check.

\section{Overall compliance for composites of aligned anisotropic phases}

We next examine the case of bimaterial composites with aligned anisotropy. The corresponding quantities in two material phases I and II are labelled by subscripts I and II. For a planar particulate composite (such as the cross-section of a fibrereinforced composite), we label the matrix as phase I and the inclusions as phase II. The term 'aligned anisotropy' states that the two material phases share the same anisotropy, but with different isotropic responses. Their respective compliance can be described as follows:

$$
\boldsymbol{S}_{\mathrm{I}, \mathrm{II}}=\left(1 / E_{\mathrm{I}, \mathrm{II}}\right)\left\{\boldsymbol{I}-\nu_{\mathrm{I}, \mathrm{II}} \boldsymbol{J}+\frac{1}{2}\left(\mathbf{1} \otimes \boldsymbol{D}_{2}+\boldsymbol{D}_{2} \otimes \mathbf{1}\right)+\boldsymbol{D}_{4}\right\} .
$$

A total of eight elastic constants are involved in the formulation of a planar composite with aligned anisotropy. The interface condition between the two material phases can 
be modelled by a constrained spring layer introduced by Moran \& Gosz (1994), in which the interfacial traction $\boldsymbol{T}$ relates to the displacement jump $[\boldsymbol{u}]$ by

$$
\left[u_{i}\right]=C_{i j} T_{j},
$$

where $C_{i j}$ is the interfacial compliance matrix. The special case of a perfectly bonded interface corresponds to a vanishing $C_{i j}$.

Concerning the overall compliance for two-dimensional particulate composites, Zheng \& Hwang (1996) developed a scheme which reduces the dependence on the elastic constants of the constituents by two. For the case of isotropic bimaterial composites, they illustrated that the compliance of a perfectly bonded composite can be determined up to a function which only involves two Dundurs parameters. Combining the orthotropic transform with their procedure, one can substantially reduce the elastic constant dependence of planar composites with aligned anisotropy. The aligned anisotropy allows us to perform a uniform coordinate transform defined in (3.9) for both material phases. In the transformed coordinates, the compliance tensors for the two material phases become

$$
\boldsymbol{S}_{\mathrm{I}, \mathrm{II}}^{*}=\left(1 / E_{\mathrm{I}, \mathrm{II}}^{*}\right)\left\{\boldsymbol{I}-\nu_{\mathrm{I}, \mathrm{II}}^{*} \boldsymbol{J}+\frac{1}{2}\left|c_{2}^{*}\right|\left(\mathbf{1} \otimes \boldsymbol{e}_{z^{*}} \otimes \boldsymbol{e}_{z^{*}}+\boldsymbol{e}_{z^{*}} \otimes \boldsymbol{e}_{z^{*}} \otimes \mathbf{1}\right)\right\},
$$

and the interfacial relation changes to

$$
\left[u_{i}^{*}\right]=C_{i j}^{*} T_{i}^{*}, \quad \text { with } \quad C_{i j}^{*}=\Omega_{p i} \Omega_{q j} C_{p q} .
$$

Under a remote traction boundary condition (which is globally equilibrium), one can write the stress invariance principle established by Moran \& Gosz (1994) in the transformed coordinates. Accordingly, the transformed stress remains invariant under the following compliance shift:

$$
\hat{\boldsymbol{S}}_{\mathrm{I}, \mathrm{II}}^{*}=k \boldsymbol{S}_{\mathrm{I}, \mathrm{II}}^{*}+\lambda \boldsymbol{J}, \quad \hat{\boldsymbol{C}}^{*}=k \boldsymbol{C}^{*}
$$

for any $\lambda$ and $k$. The strain energy stored in the composite can be evaluated over the transformed domain $\Omega^{*}$ as follows:

$$
U=\frac{1}{2} \int_{\Omega^{*}} \sigma_{i j}^{*} S_{i j k l}^{*} \sigma_{k l}^{*} \mathrm{~d} \Omega+\frac{1}{2} \sum_{\alpha} \int_{\Gamma_{\alpha}^{*}} T_{i}^{*}\left[u_{i}^{*}\right] \mathrm{d} \Gamma .
$$

Within the domain $\Omega^{*}$ composed of two material phases, the transformed compliance tensor $\boldsymbol{S}^{*}$ in (5.6) takes the value of $\boldsymbol{S}_{\mathrm{I}}^{*}$ in material phase I, and $\boldsymbol{S}_{\mathrm{II}}^{*}$ in material phase II. Substituting (5.5) into (5.6) and rearranging, one arrives at

$$
2 k U=\int_{\Omega^{*}} \sigma_{i j}^{*} \hat{S}_{i j k l}^{*} \sigma_{k l}^{*} \mathrm{~d} \Omega+\sum_{\alpha} \int_{\Gamma_{\alpha}^{*}} T_{i}^{*} \hat{C}_{i j}^{*} T_{j}^{*} \mathrm{~d} \Gamma-\lambda \int_{\Omega^{*}} \sigma_{i j}^{*} J_{i j k l} \sigma_{k l}^{*} \mathrm{~d} \Omega .
$$

Following Zheng \& Hwang (1996), the last term in (5.7) can be evaluated explicitly as

$$
-\int_{\Omega^{*}} \sigma_{i j}^{*} J_{i j k l} \sigma_{k l}^{*} \mathrm{~d} \Omega=e_{i k} \sigma_{i j}^{\infty *} \oint_{\partial \Omega^{*}} P_{k}^{*} n_{j}^{*} \mathrm{~d} s,
$$

where $\partial \Omega^{*}$ denotes the exterior boundary of the composite in the transformed coordinates.

We next evaluate the strain energy stored within the composite through macroscopically averaged quantities. Denote $\left\langle\boldsymbol{S}^{*}\right\rangle$ as the transformed compliance tensor of the composite. The strain energy stored in the composite is given by

$$
U=\frac{1}{2} A^{*} \sigma_{i j}^{\infty *}\left\langle S_{i j k l}^{*}\right\rangle \sigma_{k l}^{*},
$$

Proc. R. Soc. Lond. A (1998) 
which can be written as

$$
\begin{aligned}
2 k U & =A^{*} \sigma_{i j}^{\infty *}\left(k\left\langle S_{i j k l}^{*}\right\rangle+\lambda J_{i j k l}\right) \sigma_{k l}^{*}-\lambda A^{*} \sigma_{i j}^{\infty *} J_{i j k l} \sigma_{k l}^{\infty *} \\
& =A^{*} \sigma_{i j}^{\infty *}\left(k\left\langle S_{i j k l}^{*}\right\rangle+\lambda J_{i j k l}\right) \sigma_{k l}^{*}-\lambda e_{i k} \sigma_{i j}^{\infty *} \oint_{\partial \Omega^{*}} P_{k}^{*} n_{j}^{*} \mathrm{~d} s,
\end{aligned}
$$

where the last step is also due to Zheng \& Hwang (1996). Combining (5.7), (5.8) and (5.10), and making use of the shift relation (5.5), one has

$$
\begin{aligned}
A^{*} \sigma_{i j}^{\infty *}\left(k\left\langle S_{i j k l}^{*}\right\rangle+\lambda J_{i j k l}\right) \sigma_{k l}^{\infty *} & \\
& =\int_{\Omega^{*}} \sigma_{i j}^{*}\left(k S_{i j k l}^{*}+\lambda J_{i j k l}\right) \sigma_{k l}^{*} \mathrm{~d} \Omega+k \sum_{\alpha} \int_{\Gamma_{\alpha}^{*}} T_{i}^{*} C_{i j}^{*} T_{j}^{*} \mathrm{~d} \Gamma .
\end{aligned}
$$

The linearity of the problem renders the right-hand side of (5.11) a quadratic form of $\sigma_{i j}^{\infty *}$. One then concludes that

$$
k\left\langle\boldsymbol{S}^{*}\right\rangle+\lambda \boldsymbol{J}=\boldsymbol{K}\left(k \boldsymbol{S}_{\mathrm{I}}^{*}+\lambda \boldsymbol{J}, k \boldsymbol{S}_{\mathrm{II}}^{*}+\lambda \boldsymbol{J}, k \boldsymbol{C}^{*}, g^{*}\right),
$$

where $g^{*}$ symbolizes the dependence on the geometry of inclusion shapes in the transformed coordinates. Again, denote the difference between the overall compliance and the matrix compliance by a fourth-order tensor $\boldsymbol{H}$, which symbolizes the compliance change due to the presence of inclusions. In the transformed coordinates, the above definition becomes

$$
\left\langle\boldsymbol{S}^{*}\right\rangle=\boldsymbol{S}_{\mathrm{I}}^{*}+\boldsymbol{H}^{*} .
$$

Relations (5.12) and (5.13) prescribe the following dependence of $\boldsymbol{H}^{*}$ :

$$
k \boldsymbol{H}^{*}=\hat{\boldsymbol{K}}\left(k \boldsymbol{S}_{\mathrm{I}}^{*}+\lambda \boldsymbol{J}, k \boldsymbol{S}_{\mathrm{II}}^{*}+\lambda \boldsymbol{J}, k \boldsymbol{C}^{*}, g^{*}\right) .
$$

Let us arbitrarily take $k=E_{\mathrm{I}}^{*}$ and $\lambda=\nu_{\mathrm{I}}^{*}$, then (5.14) is reduced to

$$
E_{\mathrm{I}}^{*} \boldsymbol{H}^{*}=\tilde{\boldsymbol{K}}\left(\frac{E_{\mathrm{I}}^{*}}{E_{\mathrm{II}}^{*}}, \nu_{\mathrm{II}}^{*}-\nu_{\mathrm{I}}^{*} \frac{E_{\mathrm{II}}^{*}}{E_{\mathrm{I}}^{*}},\left|c_{2}^{*}\right|, E_{\mathrm{I}}^{*} \boldsymbol{C}^{*}, g^{*}\right)=\breve{\boldsymbol{K}}\left(\alpha^{*}, \beta^{*},\left|c_{2}^{*}\right|, E_{\mathrm{I}}^{*} \boldsymbol{C}^{*}, g^{*}\right),
$$

where $\alpha^{*}$ and $\beta^{*}$ are Dundurs parameters of the bimaterial in the transformed coordinates, and they can be evaluated from $E_{\mathrm{I}}^{*}, E_{\mathrm{II}}^{*}, \nu_{\mathrm{I}}^{*}, \nu_{\mathrm{II}}^{*}$ by the conventional Dundurs parameter formula. For the special case of perfectly bonded interfaces, the dependence on $E_{\mathrm{I}}^{*} \boldsymbol{C}^{*}$ drops out from (5.15). Moreover, if the tensor $\boldsymbol{H}$ depends on the inclusion geometry only through the area fraction $f$ of the inclusion, the relation (5.15) can be reduced to

$$
E_{\mathrm{I}}^{*} \boldsymbol{H}^{*}=\breve{\boldsymbol{K}}\left(\alpha^{*}, \beta^{*},\left|c_{2}^{*}\right|, f\right) .
$$

In the transformed coordinates, the inclusion induced compliance change for composites of aligned anisotropy, when multiplied by $E_{\mathrm{I}}^{*}$, only depends on $\left|c_{2}^{*}\right|$ and two Dundurs parameters. For the special case of rigid inclusions, (5.16) is further reduced to

$$
E_{\mathrm{I}}^{*} \boldsymbol{H}^{*}=\widehat{\boldsymbol{K}}\left(\nu_{\mathrm{I}}^{*},\left|c_{2}^{*}\right|, f\right)
$$

Combining (5.13) and (5.16) and transforming back, one finds the overall compliance tensor in the $x_{1}-x_{2}$ coordinates, in a form similar to (4.9).

Proc. R. Soc. Lond. A (1998) 


\section{Layered materials of aligned anisotropy}

This section includes another application of orthotropic transform concerning straight interfaces between dissimilar materials of aligned anisotropy. It provides a framework for interfacial fracture and for the stress analysis of layered materials. The compliances of two material phases are given in (5.1) in the $x_{1}-x_{2}$ coordinates. For the case of a perfectly bonded bimaterial interface, the interfacial compliance matrix in (5.2) vanishes; while its inverse vanishes along crack surfaces for the case of interfacial fracture. Ting (1995) explored the stress analysis of anisotropic bimaterials by a Stroh formulation. He was able to reduce the dependence of elastic constants by two, up to the capacity of a CLM shift. For the case of dissimilar materials with aligned anisotropy, the stress analysis still involves six combinative material constants under Ting's approach. This section will introduce a configuration invariant transform, through which the stress analysis on the same configuration will only depend on four combinative material constants.

Let us explore a transform that does not alter the interface configuration. Without loss of generality, a straight interface is described by $x_{2}=h$ in the original coordinates. It is mapped to an identical interface configuration (same location and orientation) $\eta_{2}=h$ in the $\eta_{1}-\eta_{2}$ coordinates defined by

$$
\eta_{i}=\tilde{\Omega}_{i j} x_{j}, \quad \text { with } \quad\left[\tilde{\Omega}_{i j}\right]=\left[\begin{array}{cc}
\frac{1+\gamma \bar{\gamma}+2 \gamma_{1}}{1-\gamma_{1}^{2}+\gamma_{2}^{2}} & \frac{-2 \gamma_{1} \gamma_{2}}{1-\gamma_{1}^{2}+\gamma_{2}^{2}} \\
0 & 1
\end{array}\right] .
$$

The transform (6.1) describes stretching and shearing parallel to the $x_{1}$-direction, and leaves any bimaterial configurations (which may have many layers) with straight interfaces parallel to the $x_{1}$-axis undistorted. In the $\eta_{1}-\eta_{2}$ coordinates, however, the governing equation (in the material phases above and below the interface) is given by

$$
\left\{\frac{\partial^{2}}{\partial \eta_{1}^{2}}+\frac{\partial^{2}}{\partial \eta_{2}^{2}}-\left|c_{2}^{*}\right| \cos 2 \varphi\left(\frac{\partial^{2}}{\partial \eta_{1}^{2}}-\frac{\partial^{2}}{\partial \eta_{2}^{2}}\right)-2\left|c_{2}^{*}\right| \sin 2 \varphi \frac{\partial^{2}}{\partial \eta_{1} \eta_{2}}\right\}\left(\frac{\partial^{2} \Phi}{\partial \eta_{1}^{2}}+\frac{\partial^{2} \Phi}{\partial \eta_{2}^{2}}\right)=0
$$

where

$$
\varphi=\arctan \left(\Omega_{21} / \Omega_{11}\right)=\arg (1+\gamma)+\omega=\arg (1+\gamma)-\frac{1}{2} \arg \tilde{c}_{2}
$$

denotes the rotation angle of the interface in the $z^{*}$ coordinates. Equation (6.2) corresponds to the governing equation of an orthotropic material whose axis of orthotropy forms an angle $\varphi$ with the interface. It was rigorously proved by Ting (1995) via Stroh formalism that the stress analysis for general anisotropic bimaterials only depends on two Dundurs parameters, as well as all eigenvalues of two material phases. The latter are irrelevant to the isotropic parameters of the two material phases. Since the bimaterial has aligned anisotropy, Ting's analysis suggests that the stress response in the $\eta_{1}-\eta_{2}$-plane only depends on two Dundurs parameters and the eigenvalues of (6.2). Take a solution of (6.2) in the form of

$$
\Phi\left(\eta_{1}, \eta_{2}\right)=\Phi\left(\eta_{1}+p \eta_{2}\right),
$$

where $p$ is the eigenvalue of the problem. Substituting (6.4) into (6.2), one has the following eigenvalue equation:

$$
\left\{1-\left|c_{2}^{*}\right| \cos 2 \varphi-2 p\left|c_{2}^{*}\right| \sin 2 \varphi+p^{2}\left(1+\left|c_{2}^{*}\right| \cos 2 \varphi\right)\right\}\left(1+p^{2}\right)=0 .
$$

Proc. R. Soc. Lond. A (1998) 
The four eigenvalues are given by

$$
p= \pm \mathrm{i}, \quad p=\frac{\left|c_{2}^{*}\right| \sin 2 \varphi \pm \mathrm{i} \sqrt{1-\left|c_{2}^{*}\right|^{2}}}{1+\left|c_{2}^{*}\right| \cos 2 \varphi} .
$$

They depend only on the values of $\left|c_{2}^{*}\right|$ and $\varphi$. The configuration invariant transform (6.1) enables us to claim that the stress analysis for layered materials of aligned anisotropy depends on four combinative material constants: two Dundurs parameters, $\left|c_{2}^{*}\right|$ and $\varphi$.

\section{Concluding remarks}

The orthotropic transform introduced in this paper substantially reduces the dependence of elastic constants in planar anisotropic solids. The reduction is quite helpful in estimating the overall material response, especially when the overall response is insensitive to the geometry of the second phase (voids or inclusions). In combination with the CLM shift (which removes the dependence of two isotropic material constants), the orthotropic transform scheme outlined here can eliminate the dependences of five elastic constants. If the overall estimate is rather sensitive to the geometry, or if detailed stress analysis is required, the effectiveness of orthotropic transform may be shadowed. The orthotropic transform sometimes complicates the geometry (such as transforming a circle to an ellipse), sometimes simplifies the geometry (such as transforming an ellipse to a circle), and sometimes leads to equivalent geometry (such as transforming a layered configuration to another layered configuration). There are a spectrum of geometry-dependent problems that can be simplified by proper orthotropic transform.

The authors acknowledge the financial support from the National Science Council, through grant NSC86-2212-E002-008, which makes the present research collaboration possible. Stimulating discussions with Professor Q.-S. Zheng are appreciated.

\section{References}

Cherkaev, A., Lurie, K. \& Milton, G. W. 1992 Invariant properties of stress in plane elasticity and equivalent classes of composites. Proc. R. Soc. Lond. A 438, 519-529.

Chiang, Q. T. 1994 Correspondences and related analyses between in-plane anisotropic and isotropic elasticity. Master thesis, Department of Mechanical Engineering, National Taiwan University.

Christensen, R. M. 1993 Effective properties of composite materials containing holes. Proc. $R$. Soc. Lond. A 440, 461-473.

Day, A. R., Snyder, K. A., Garboczi, E. J. \& Thorpe, M. F. 1992 The elastic moduli of a sheet containing circular holes. J. Mech. Phys. Solids 40, 1031-1051.

Dundurs, J. \& Markenscoff, X. 1993 Invariance of stresses under a change in elastic compliances. Proc. R. Soc. Lond. A 443, 289-300.

Green, A. E. \& Zerna, W. 1960 Theoretical elasticity. Oxford University Press.

Moran, B. \& Gosz, M. 1994 Stress invariance in plane anisotropic elasticity. Modelling Simul. Mater. Sci. Engng 2, 677-688.

Thorpe, M. F. \& Jasiuk, I. 1992 New results in the theory of elasticity for two-dimensional composites. Proc. R. Soc. Lond. A 438, 531-544.

Ting, T. C. T. 1995 Generalized Dundurs constants for anisotropic bimaterials. Int. J. Solids Struct. 32, 483-500.

Proc. R. Soc. Lond. A (1998) 
Yang, W., Suo, Z. \& Shih, C. F. 1991 Mechanics of dynamic debonding. Proc. R. Soc. Lond. A 433, 679-697.

Zheng, Q.-S. \& Hwang, K. C. 1996 Reduced dependence of defect compliance on matrix and inclusion elastic properties in two-dimensional elasticity. Proc. R. Soc. Lond. A 452, 24932503.

Zheng, Q.-S. \& Hwang, K. C. 1997 Two-dimensional elastic compliances of materials with holes and microcracks. Proc. R. Soc. Lond. A 453, 353-364.

Proc. R. Soc. Lond. A (1998) 
